\title{
The Upgrade and Performance of the ATLAS Tau Trigger towards Run 2
}

\author{
Andrew Karamaoun ${ }^{* *}$ \\ on behalf of the ATLAS Collaboration \\ E-mail: andrew.karamaoun@cern.ch
}

Tau triggers are used in a variety of highly important ATLAS physics analyses, including the measurement of the Higgs boson coupling to fermions and searches for Higgs boson partners or heavy resonances decaying into pairs of tau leptons. As proton-proton collisions at the LHC reach instantaneous luminosities of over $10^{34} \mathrm{~cm}^{-2} \mathrm{~s}^{-1}$ in Run 2, the strategies for triggering have become more sophisticated than in Run 1. In these conditions tau lepton triggers suffer from severe rate limitations, despite the advancements in algorithms used in the tau identification. New fast algorithms and the addition of topological selections are the main developments introduced to maintain the tau trigger sensitivity needed to support the large program of tau lepton physics analyses. In Run 2, topological criteria can be applied already at the first trigger level, due to the addition of the L1 topological processor. This makes it possible to use detailed information from sub-detectors in order to apply real-time event topology cuts. The evolution of the ATLAS tau trigger and its performance will be presented, including initial results from the early days of the LHC Run 2 operation.

The European Physical Society Conference on High Energy Physics 22-29 July 2015

Vienna, Austria

* Speaker.

${ }^{\dagger}$ University of Alberta 


\section{Introduction}

ATLAS [1] is a general purpose detector placed at one of the interaction points of the Large Hadron Collider (LHC), a particle accelerator that collides two opposing beams of protons at a design centre-of-mass energy, $\sqrt{s}$, of $14 \mathrm{TeV}$. The collision data taking periods that occured between the years 2009 to 2013 are referred to as Run 1, and experienced collisions occuring at up to $\sqrt{s}=8 \mathrm{TeV}$ and an instantaneous luminosity of $8 \times 10^{33} \mathrm{~cm}^{-2} \mathrm{~s}^{-1}$. Since May 2015 (Run 2), the LHC operates at a $\sqrt{s}=13 \mathrm{TeV}$ and has a target instantaneous luminosity of $2 \times 10^{34} \mathrm{~cm}^{-2} \mathrm{~s}^{-1}$. In order to select and analyze interesting events in a timely manner, while considering storage constraints, a system called a trigger is used to decide which events to accept and which to reject. Since Run 2, the trigger is divided into two parts: Level-1 (L1) and the High-Level-Trigger (HLT). L1 is the first to obtain information about an event, is hardware based, takes less than $2.5 \mu \mathrm{s}$ to make a decision, and has a maximum event acceptance rate of about $100 \mathrm{kHz}$. The $\mathrm{L} 1$ determines regions of interest (RoIs) in the $\eta, \phi^{1}$ space of the detector based on energy readouts from the calorimeter and signals from muon spectrometers, and passes this information on to the next level of the trigger. Using data from all detectors, the HLT obtains more information about the RoIs obtained from L1, and determines, in about $0.2 \mathrm{~s}$, whether to accept or reject the event. It is software-based and has an event ouput frequency of about $1 \mathrm{kHz}$. The HLT is the result of merging the Level-2 (L2) and Event-Filter (EF) stages of Run 1. Figure 1 shows an overview of the ATLAS trigger and data acquisition system for Run 2 [2].
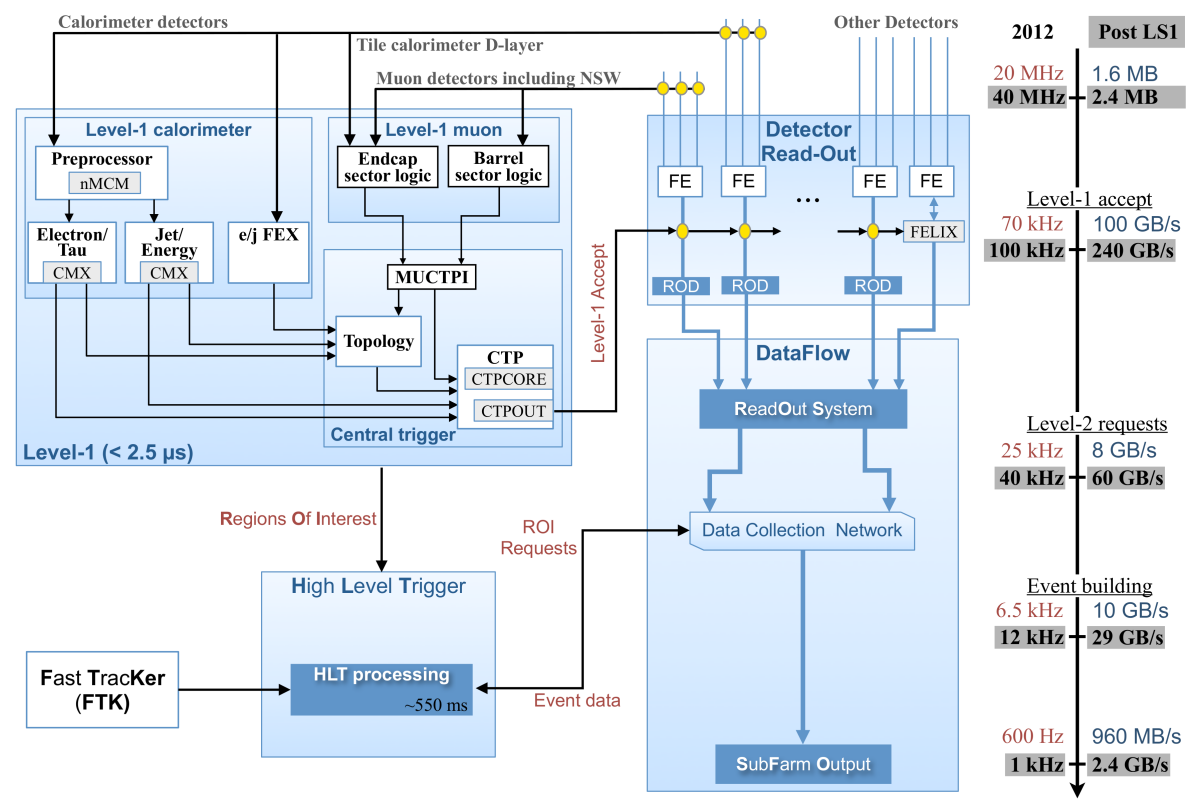

Figure 1: A schematic overview of the trigger and data acquisition system for Run 2. A comparison between the Run 1 rates and bandwith usage is also given. Taken from Ref. [2].

\footnotetext{
${ }^{1}$ ATLAS uses a right-handed coordinate system with its origin at the nominal interaction point in the center of the detector and the $\mathrm{z}$-axis along the beam pipe. Cylindrical coordinates $(r, \phi)$ are used in the transverse plane, $\phi$ being the azimuthal angle around the beam pipe. The pseudorapidity $\eta$ is defined in terms of the polar angle $\theta$ by $\eta:=\ln [\tan (\theta / 2)]$, and the distance $\Delta R$ in $\eta, \phi$ space is defined as $\Delta R=\sqrt{(\Delta \eta)^{2}+(\Delta \phi)^{2}}$.
} 


\section{The Tau Lepton}

The tau is the heaviest lepton with a mass of $1.78 \mathrm{GeV}$ and is the only one that can decay hadronically [3]. Due to its short lifetime, it decays inside the beam pipe of the LHC. It has a $35 \%$ branching fraction for leptonic decays and $65 \%$ for hadronic ones, $\tau(\mathrm{had})$, where the hadronic decay products mostly consist of pions and neutrinos. The neutral pions decay into photons before reaching the detector and thus leave an electromagnetic (EM) shower in the EM calorimeter. The charged pions lose a significant fraction of their energy in the hadronic (HAD) calorimeter, where they leave a hadronic shower of energy deposits.

Taus play an important role in both searches for beyond-standard-model (BSM) phenomena, such as supersymmetry [4], and precision studies of the standard model (SM), such as the measurement of the coupling of the Higgs boson [5] to the leptons. Due to their mass, they couple strongly to both the SM and BSM Higgs bosons, and in some supersymmetric (SUSY) scenarios, are predicted to have the lightest supersymmetric partners of the leptons. Figure 2 shows Feynman diagrams for charged Higgs production with a decay into a tau lepton as well as chargino-neutralino production that results in the production of stau pairs that decay into taus plus missing transverse momentum. The charged Higgs boson is predicted by any model with two Higgs doublets, such as SUSY, and the charginos and neutralinos are the superpartners of the gauge bosons, as also predicted by SUSY.
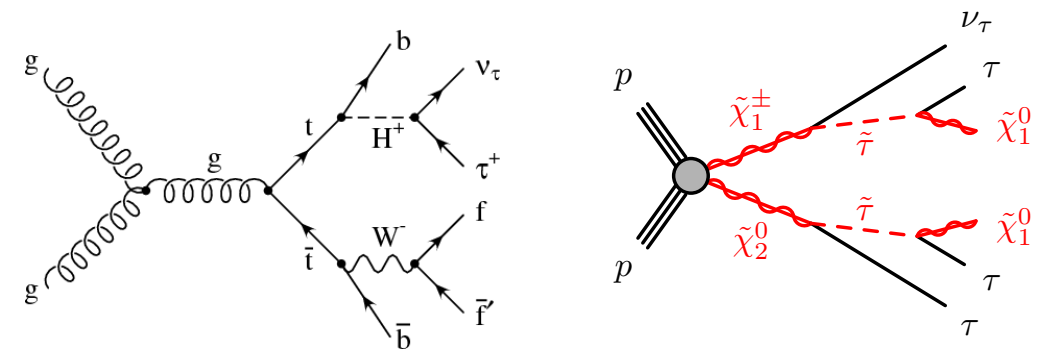

Figure 2: Feynman diagrams for charged Higgs production with a decay into a tau lepton (left) and for chargino-neutralino production with taus in the decay product (right).

\section{The Tau Trigger}

The ATLAS tau trigger [6] is designed to select hadronically decaying taus, while the electron and muon triggers are used for the leptonic decay channels. In Run 2, the single tau trigger item requires $p_{\mathrm{T}}>125 \mathrm{GeV}$. All other tau triggers use multi-object combinations to achieve lower thresholds. Table 1 shows the combined tau triggers together with their corresponding kinematic requirements and examples of targeted physics processes.

QCD induced jets form the dominant reducible background for taus. Hadronic tau jets tend to have a lower track multiplicity, are well collimated, and have a displaced vertex due to their lifetime. Figure 3 illustrates the difference between the signature of a jet originating from a gluon and one originating from a tau. Compared to the other elementary particles detected by ATLAS, the irreducible background is small for BSM signatures involving taus, and mainly comes from the $W \rightarrow \tau \nu$ and $Z \rightarrow \tau \tau$ channels of production. 


\section{Process}

$H_{\mathrm{SM}} \rightarrow \tau_{\text {had }} \tau_{\text {had }}$
$H_{\mathrm{SM}} \rightarrow \tau_{\text {had }} \tau_{\text {lep }}$
or $Z \rightarrow \tau_{\text {had }} \tau_{\text {lep }}$
$\tilde{\chi}_{1}^{ \pm} \tilde{\chi}_{2}^{0} \rightarrow \tau^{ \pm} \tau^{\mp} \tau^{ \pm} v \tilde{\chi}_{1}^{0} \tilde{\chi}_{1}^{0}$
or $\tilde{\chi}_{1}^{ \pm} \tilde{\chi}_{1}^{ \pm} \rightarrow 2 \times \tau^{ \pm} v \tilde{\chi}_{1}^{0}$
or $\tilde{\tau}^{ \pm} \tilde{\tau}^{\mp} \rightarrow \tau^{+} \tau^{-} \tilde{\chi}_{1}^{0} \tilde{\chi}_{1}^{0}$
$W^{\prime} \rightarrow \tau v$

Trigger

$$
\begin{aligned}
& \tau_{\text {had-vis }}+\tau_{\text {had-vis }} \\
& \tau_{\text {had-vis }}+e \\
& \tau_{\text {had-vis }}+\mu \\
& \tau_{\text {had-vis }}+\tau_{\text {had-vis }}+p_{\mathrm{T}}^{\text {miss }} \\
& \tau_{\text {had-vis }}
\end{aligned}
$$

$$
\begin{aligned}
& \text { Requirements at HLT [GeV] } \\
& p_{\mathrm{T}}\left(\tau_{1}\right)>25 \& p_{\mathrm{T}}\left(\tau_{2}\right)>35 \\
& p_{\mathrm{T}}(\tau)>25 \& p_{\mathrm{T}}(e)>17 \\
& p_{\mathrm{T}}(\tau)>25 \& p_{\mathrm{T}}(\mu)>14 \\
& p_{\mathrm{T}}\left(\tau_{1}\right)>25 \& p_{\mathrm{T}}\left(\tau_{2}\right)>35 \\
& \& p_{\mathrm{T}}^{\text {miss }}>50 \\
& p_{\mathrm{T}}(\tau)>125
\end{aligned}
$$

Table 1: Tau triggers with their corresponding kinematic requirements. For each trigger, at least one example of a targetted physics process is given. 'had' refers to hadronic, 'lep' to leptonic, and 'vis' to visible tau decays, emphasizing that the neutrino components of the tau decay are not visible.
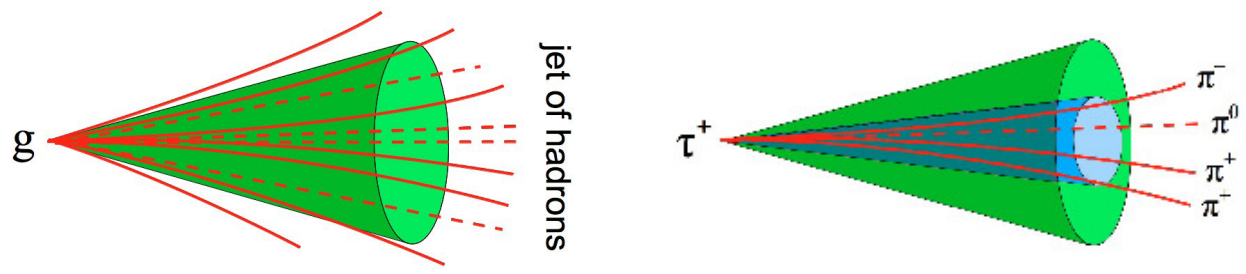

Figure 3: A comparison between the signature of a jet originating from a gluon (left) and one originating from a tau lepton (right).

For L1, the tau trigger selects RoIs based on the energy deposited in $\Delta \eta \times \Delta \phi=0.1 \times 0.1$ regions (towers) within the EM calorimeter. The energy within a core region of $2 \times 2$ towers must surpass a minimum threshold and the energy in a ring of towers (isolation region) surrounding the core region must be less than a maximum threshold. The isolation requirement is used to supress the jet background. In Run 2, the isolation energy threshold is a function of the core energy threshold, which allows for stable performance across the entire range of energies. Furthermore, topological selections will be applied. Once the instantaneous luminosity becomes sufficiently high, a requirement of $\Delta R<2.8$ between the two taus of the ditau trigger will be placed to ensure sufficiently low acceptance rates. The topological trigger is a new hardware component of the L1 ATLAS trigger system, and for taus, has been optimized for SM Higgs physics, such that an approximately 95\% acceptance efficiency for two taus decaying from the Higgs boson is expected.

The HLT begins by precisely reconstructing the energy of each tau candidate with a technique known as topoclustering [7]. In Run 2, the calculation has been made more precise by using the same topoclustering algorithm as used in the offline ${ }^{2}$ reconstruction. With this information, the trigger rejects all RoIs that fail to surpass a certain transverse energy threshold. Next, the fast tracking algorithm is executed, which calculates the number of tracks in the core and isolation regions for an RoI, using a simple extrapolation seeded from pairs of inner detector hits [8]. RoIs without any or with more than three core tracks are rejected, as are RoIs with more than one isolated track. Once those steps have been completed, the rate of events is reduced by a factor of about 10 , and this allows enough time to perform the precision track reconstruction, nearly equivalent to

\footnotetext{
${ }^{2}$ The processing of events that have not yet been accepted by a trigger is said to be done online. If the events have been accepted by a trigger, the processing is said be done offline.
} 
that used offline. All detector inputs are then combined to calculate 13 variables that are derived from both calorimeter and tracking information and are fed into a boosted-decision-tree (BDT) discriminant that is nearly equivalent to the one used offline. The variables are linearly corrected as a function of the number of simultaneous collisions, and the discriminant is $p_{\mathrm{T}}$ flattened, so that the discriminant is minimally dependent on the pile-up conditions and transverse momentum of the candidate.

Figure 4 shows a summary of the steps involved in the tau trigger selection process.

Level 1

High Level Trigger

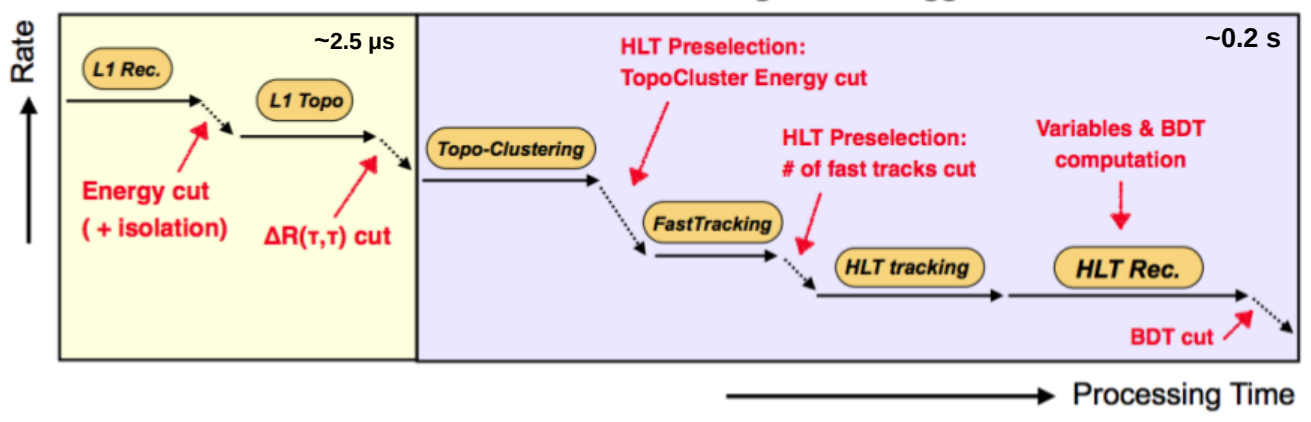

Figure 4: A summary of the steps involved in the tau trigger selection process.

\section{Performance}

The performance of the tau trigger can be studied with the tag-and-probe technique using a sample enriched with hadronic taus by selecting events from the $Z \rightarrow \tau \tau \rightarrow \mu \tau$ (had) channel. [9] The sample is obtained by selecting events accepted by the muon trigger and with requirements on offline reconstructed objects such as the presence of a well-reconstructed muon, small transverse mass $^{3}$, and a visible mass compatible with that of the $\mathrm{Z}$ boson. The requirements are used to tag events from this channel and reduce backgrounds from other processes. Since the muon trigger is used, the hadronic tau is then an unbiased probe of the tau trigger performance.

Figure 5 shows the distributions of several kinematic variables for tau candidates passing the HLT tau trigger with a transverse momentum threshold of $25 \mathrm{GeV}$ and the medium online identification requirement ${ }^{4}$, using early $13 \mathrm{TeV}$ data. The tau candidates are observed in an enriched sample of $Z \rightarrow \tau \tau \rightarrow \mu \tau(\mathrm{had})$ events and are considered if matched to a tau candidate passing the medium offline tau identification criteria. The data is compared to signal $Z \rightarrow \tau \tau$ simulated events and a combined background consisting of $Z \rightarrow l l, \mathrm{~W}+\mathrm{jets}$, top events, and a data driven multi-jet estimate.

As a cross-check, the distributions of these kinematic variables from a sample of misidentified tau candidates is examined. Figure 6 shows the $p_{\mathrm{T}}$ and $\eta$ distributions of the HLT tau candidates in an enriched sample of $W \rightarrow \mu v$ events, where the taus are likely to be jets originating from quarks

\footnotetext{
${ }^{3} m_{\mathrm{T}}:=\sqrt{2 p_{\mathrm{T}}^{\ell} E_{\mathrm{T}}^{\text {miss }}\left(1-\cos \Delta \phi\left(\ell, E_{\mathrm{T}}^{\text {miss }}\right)\right)}$

${ }^{4}$ The online identification requirement that has an acceptance efficiency of $70 \%$ for a signal $Z \rightarrow \tau \tau$ simulation sample designed for online identification optimization. The efficiency is defined as the number of tau candidates that pass the requirement divided by the number of tau candidates that pass only the $\mathrm{L} 1$ and $p_{\mathrm{T}}$ requirements of the trigger.
} 

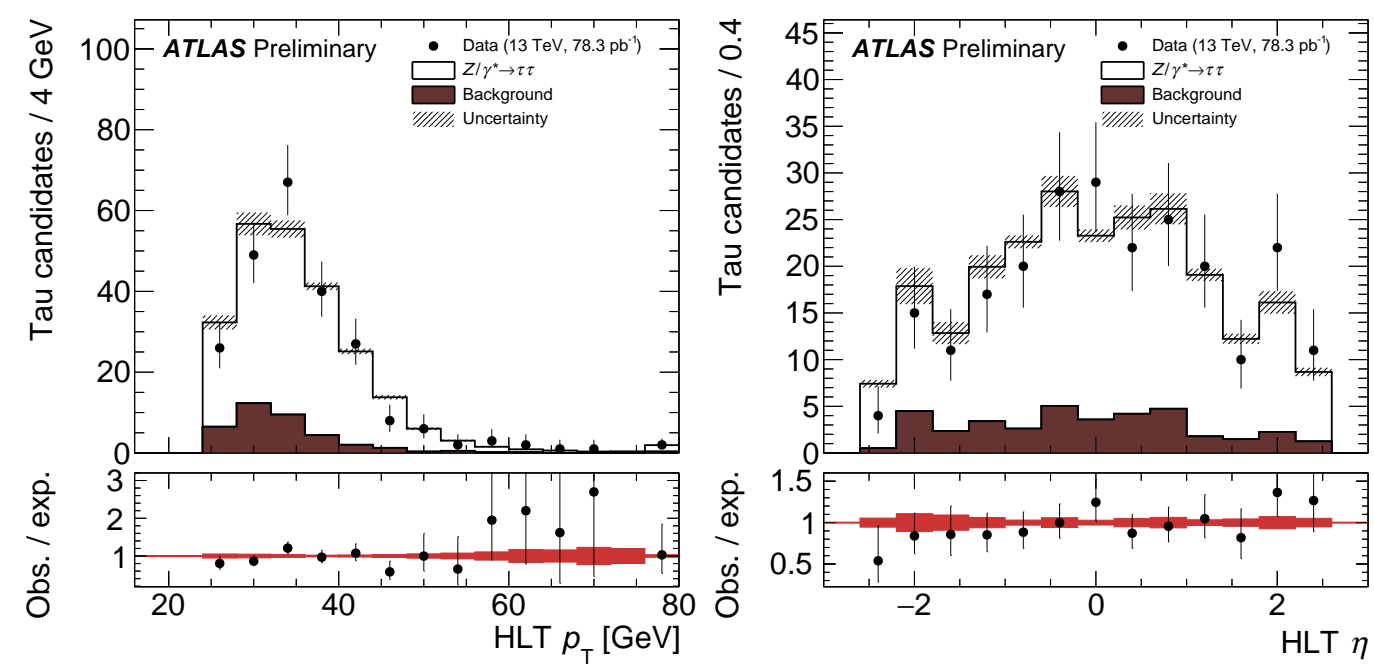

Figure 5: The distributions of the transverse momentum (left) and pseudorapidity (right) for tau candidates passing the HLT tau trigger with a transverse momentum threshold of $25 \mathrm{GeV}$ and the medium online identification requirement, using $13 \mathrm{TeV}$ data taken in Summer 2015, with a $50 \mathrm{~ns}$ bunch spacing. The tau candidates are observed in an enriched sample of $Z \rightarrow \tau \tau \rightarrow \mu \tau(\mathrm{had})$ events and are considered if matched to a tau candidate passing the medium offline tau identification criteria. Taken from Ref. [10].

or gluons that are reconstructed as tau jets and accepted by the tau trigger. The data is compared to signal $W \rightarrow \mu v+$ jets simulated events and a combined background consisting of $Z \rightarrow \tau \tau, Z \rightarrow l l$, top events, and a data driven multi-jet estimate. The former is the $\mathrm{W}+$ jets background considered in Figure 5 and is a major background for both the tag-and-probe study and several key physics searches involving tau signatures.

Finally, the efficiency of the tau trigger with respect to the offline tau reconstruction can be measured from the $Z \rightarrow \mu \tau(\mathrm{had})$ sample obtained from the tag-and-probe method. By definition, the efficiency is the number of offline identified taus passing the trigger criteria divided by the total number of offline identified taus, after subtraction of the backgrounds. Figure 7 shows the L1 and combined L1-HLT efficiencies and a comparison with $Z \rightarrow \tau \tau$ simulation.

\section{Conclusion}

An overview of the current state of the ATLAS tau trigger was given. Upgrades for Run 2 include revised transverse momentum thresholds, combined triggers, improved isolation energy thresholds, topological L1 requirements and improved topo clustering at the HLT. The upgrades were necessary in order to keep the same physics sensitivity under the more demanding Run 2 conditions. Using early Run 2 data, the performance of the tau trigger was studied using the tagand-probe method involving the $Z \rightarrow \tau \mu$ and $W \rightarrow \mu v$ channels. Even with the significantly higher energy conditions, the tau trigger efficiency shows good data-simulation agreement and is larger than $90 \%$ in the transverse momentum plateau region. 

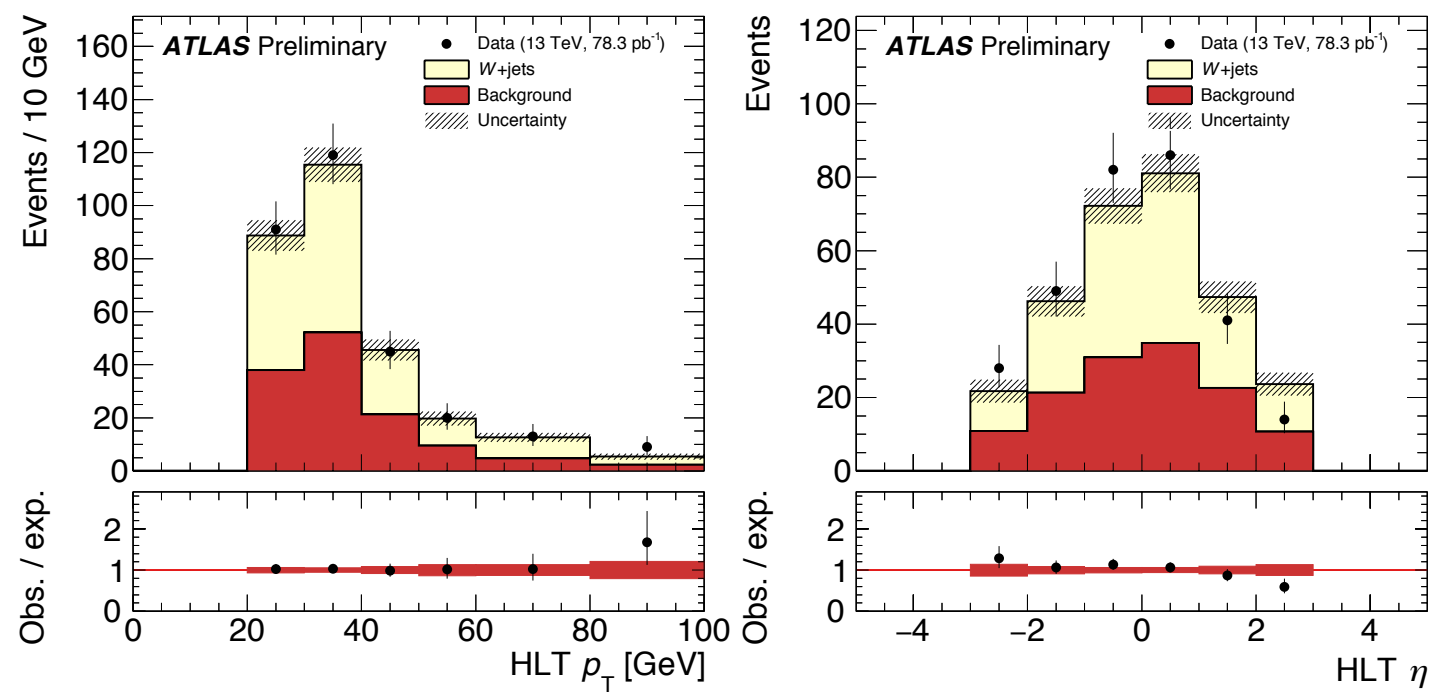

Figure 6: The transverse momentum (left) and pseudorapidity (right) distributions of HLT tau candidates in an enriched sample of $W \rightarrow \mu v$ events, where the taus are likely to be jets originating from quarks or gluons that are reconstructed as tau jets and accepted by the tau trigger. The transverse momentum threshold of the trigger is $25 \mathrm{GeV}$. Taken from Ref. [10].
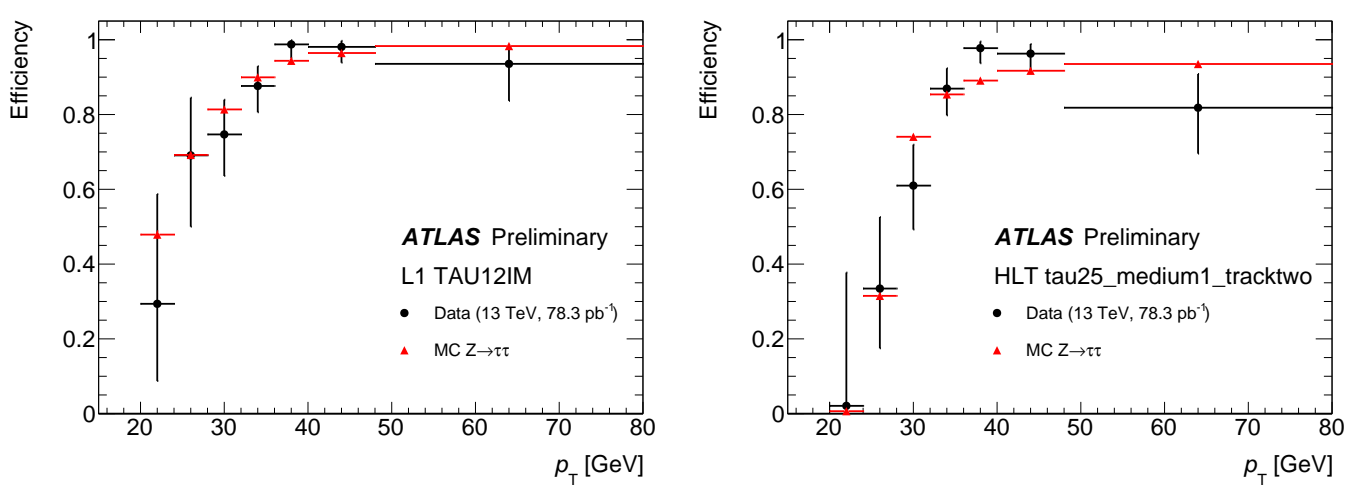

Figure 7: The L1 (left) and combined L1-HLT (right) tau trigger efficiencies measured in data and compared to simulation, with respect to offline reconstructed tau candidates with transverse momentum above $20 \mathrm{GeV}$, one or three tracks and passing the medium offline identification criteria. The data consists of an enriched sample of $Z \rightarrow \tau \tau \rightarrow \mu \tau$ (had) events taken from $13 \mathrm{TeV}$ data collected in Summer 2015, with a $50 \mathrm{~ns}$ bunch spacing. The $\mathrm{L} 1$ trigger has a $12 \mathrm{GeV}$ transverse energy threshold and the medium isolation criteria. The HLT trigger requires candidates to have at least $25 \mathrm{GeV}$ of transverse momentum, between one and three tracks and the medium online identification requirement. Taken from Ref. [10]. 


\section{References}

[1] ATLAS Collaboration, The ATLAS Experiment at the CERN Large Hadron Collider, JINST 3 (2008) S08003.

[2] R. Bartoldus et. al., Technical Design Report for the Phase-I Upgrade of the ATLAS TDAQ System, CERN, Geneva, CERN-LHCC-2013-018 (2013), http://cds.cern.ch/record/1602235.

[3] K. A. Olive et al. [Particle Data Group Collaboration], Review of Particle Physics, Chin. Phys. C 38 (2014) 090001.

[4] ATLAS Collaboration, Search for the direct production of charginos, neutralinos and staus in final states with at least two hadronically decaying taus and missing transverse momentum in pp collisions at $\sqrt{s}=8 \mathrm{TeV}$ with the ATLAS detector, J. High Energy Phys. 10 (2014) 96 [arXiv:1407.0350].

[5] ATLAS Collaboration, Search for the Standard Model Higgs boson in the $H \rightarrow \tau^{+} \tau^{-}$decay mode in $s \sqrt{s}=7 \mathrm{TeV}$ pp collisions with ATLAS, J. High Energy Phys. 09 (2012) 70 [arXiv:1206.5971].

[6] ATLAS Collaboration, Identification and energy calibration of hadronically decaying tau leptons with the ATLAS experiment in $p p$ collisions at $\sqrt{s}=8 \mathrm{TeV}$, Eur. Phys. J. C75 (2015) 303 [arXiv:1412.7086].

[7] W. Lampl et. al., Calorimeter Clustering Algorithms: Description and Performance, CERN, Geneva, ATL-LARG-PUB-2008-002 (2008), http://cds.cern.ch/record/1099735.

[8] ATLAS Collaboration, Performance of the ATLAS Trigger System in 2010, Eur. Phys. J. C 72 (2012) 1849 [arXiv:1110.1530].

[9] ATLAS Collaboration, Commissioning of the reconstruction of hadronic tau lepton decays in ATLAS using pp collisions at $\sqrt{s}=13 \mathrm{TeV}$, CERN, Geneva, ATL-PHYS-PUB-2015-025 (2015), http://cds.cern.ch/record/2037716.

[10] ATLAS Collaboration, Public Tau Trigger Plots for Collision Data, CERN, Geneva, http://twiki.cern.ch/twiki/bin/view/AtlasPublic/TauTriggerPublicResults. 